\title{
New pediatric risk factors for amblyopia: strabismic versus refractive
}

\author{
Sandra Guimaraes ${ }^{1-3}$, Maria Vieira ${ }^{4}$, Tatiana Queirós ${ }^{1}$, Andreia Soares ${ }^{1}$, Patrício Costa $^{2,3,5}$, Eduardo Silva ${ }^{6-8}$ \\ ${ }^{1}$ Department of Ophthalmology, Hospital de Braga, Braga - Portugal \\ ${ }^{2}$ Life and Health Sciences Research Institute (ICVS), School of Medicine, University of Minho, Braga - Portugal \\ ${ }^{3}$ ICVS/3Bs, PT Government Associate Laboratory, Braga/Guimarães - Portugal \\ ${ }^{4}$ School of Medicine, University of Minho, Braga - Portugal \\ ${ }^{5}$ Clinical Academic Center-Braga, Braga - Portugal \\ ${ }^{6}$ Department of Ophthalmology, Centro Cirurgico de Coimbra, Coimbra - Portugal \\ ${ }^{7}$ IBILI, Faculty of Medicine, University of Coimbra, Coimbra - Portugal \\ ${ }^{8}$ Department of Ophthalmology, CHLN, Lisbon - Portugal
}

Sandra Guimaraes and Maria Vieira contributed equally to this work.

\begin{abstract}
Purpose: To assess the role of risk factors for amblyopia, such as family history and neonatal background, for the prediction of either strabismic amblyopia or refractive amblyopia.

Methods: In this retrospective case-control model, the study population included all children born at the Hospital de Braga during 1997-2012 (3 to 18 years old) with ophthalmologic consultation in 2014. Data collection was performed from the clinical database and through telephone questionnaire surveys.

Results: A total of 298 (50\%) controls and 298 (50\%) cases (120 [40.3\%] strabismic amblyopia and 178 [59.7\%] refractive amblyopia) were analyzed. A significantly lower birthweight was detected in patients with strabismic amblyopia (mean 2,961 g [95\% confidence interval (Cl) 2,827-3,096]) compared to controls (mean 3,198 g [95\% Cl $3,125-3,271])(p=0.002)$. Five-minute Apgar was significantly lower in patients with strabismic amblyopia (mean 9.57 [95\% Cl 9.37-9.77]) than in controls (mean 9.83 [95\% Cl 9.77-9.90]) $(p=0.004)$ or patients with refractive amblyopia (mean 9.79 [95\% Cl 9.69-9.89]) ( $p=0.031$ ). Family history of either amblyopia or strabismus was associated with amblyopia $\left(\chi^{2}[2, n=562]=12.66 ; p=0.002 ;\right.$ Cramer $V=0.150 ; \chi^{2}[2, n=561]=11.0 ; p=0.004 ;$ Cramer $V=0.140)$, but was significantly more associated with strabismic amblyopia $(p=0.0023$ and $p=0.0032)$ than with refractive amblyopia ( $p=0.48$ and $p=0.015$, respectively). Multinomial logistic regression model explained $50.8 \%$ of the variance in amblyopia development. Low 5-minute Apgar had a relevant odds ratio (OR) for either strabismic amblyopia (OR 3.44; $p=0.066$ ) or refractive amblyopia (OR 3.30; $p=0.077$ ).

Conclusions: This division in amblyopia subtypes gives a new perspective of the risk factors for amblyopia, with family history and some obstetrician/neonatal outcomes appearing to be more relevant in strabismic amblyopia. Educating health care providers to recognize these risk factors can result in an early ophthalmologic referral.
\end{abstract}

Keywords: Amblyogenic risk factors, Family history, Neonatal background

\section{Introduction}

Amblyopia is a neurodevelopmental vision disorder caused by sensory deficits during early life (1). It is a common ophthalmologic disorder in pediatric age, with considerably

Accepted: August 23, 2017

Published online: September 11, 2017

Corresponding author:

Sandra Guimaraes

Department of Ophthalmology

Hospital de Braga,

Braga, Portugal

sandraguimaraes.eye@gmail.com different prevalence rates according to the country and the criteria used in diagnosis (2). Since amblyopia is the effect of a pathologic process, recent studies propose to include in its diagnosis not only acuity-based criteria, but also the concomitant presence of causal factors, called amblyogenic risk factors (ARF) $(3,4)$. Functional eye anomalies, such as refractive error, strabismus, and media opacity, are established ARF (5). Amblyopia is regarded as an important public health problem as it is the main cause of vision loss in children and one of the leading causes of visual impairment in adults $(6,7)$. The prompt detection and treatment of amblyopia could prevent or cure this consequence $(8,9)$. Therefore, this pediatric ophthalmologic screening topic, in order to detect decrease in visual acuity and/or ARF, has gained widespread attention in recent decades. Also, it seems that screening high-risk 
children could be more efficient and cost-effective $(10,11)$, so further research has gradually focused on the ARF. Although some ocular conditions have already been established and validated, it is possible that amblyopic children have additional risk factors. Some personal or familial characteristics seem to be linked with amblyopia $(5,12-15)$, but there is no consensus among the scientific community as to predictive factors of amblyopia development. The neonatal background, such as the 5-minute Apgar score, is a helpful predictor of neurodevelopment and a relevant prognostic factor for infants with neonatal hypoxia (16). There is no consensus about the role of prematurity, low birthweight (LBW), neonatal hypoxia or admission to the intensive care unit (ICU) $(13-15,17)$. Family history is also controversial. In recent studies, an association with ophthalmologic history (refractive error, amblyopia, or strabismus) in relatives was revealed $(17,18)$. Current evidence concerning the ARF's predictive power is not clear. Thus, the main goal of this study was to assess the role of risk factors for amblyopia, such as family history and neonatal background, for the prediction of either strabismic amblyopia (SA) or refractive amblyopia (RA).

\section{Methods}

This was a retrospective case-control study. Study protocol and informed consent were reviewed and approved by the local ethical committee of Hospital de Braga. The study population corresponded to all children born in Hospital de Braga during 1997-2012 ( 3 to 18 years old at the time of collection) with an ophthalmologic consultation in 2014. Retrospective assess to all ophthalmologic information present in the electronic clinical database (Global Intelligence Technologies; Glintt ${ }^{\circledR}$ ) was made.

Inclusion criteria included diagnosis of amblyopia and registration of occlusive treatment in addition to strabismus diagnosis or refractive error detection. In cases without occlusive treatment, the inclusion criteria for unilateral amblyopia were as follows: an intraocular difference of $\geq 2$ lines in best-corrected visual acuity (BCVA) and an amblyogenic refractive error (ARE) (ARE defined as follows: myopia if sphere $\geq-3 \mathrm{D}$; hyperopia if sphere $\geq+1.50 \mathrm{D}$; or astigmatism if cylinder $\geq \pm 2$ D) (19). For bilateral amblyopia, the criteria were defined as ARE present plus bilateral BCVA $\leq 6 / 10$ in 3- or 4-year-old subjects or ARE plus bilateral BCVA $\leq 8 / 10$ in 5+-year-old subjects. Simultaneous SA and RA were categorized as strabismic amblyopia. Exclusion criteria included organic amblyopia, BCVA less than normal without ARE, or normal BCVA with ARE. All selected cases were reviewed by a trained ophthalmologist. The controls were selected among the study population, according to the exclusion criteria, stratified by age and sex.

Telephone questionnaire surveys, which included questions related to ophthalmologic and obstetrician/neonatal data, were applied in a blinded way, with a response rate of $94.5 \%$. The questionnaire began in accordance with the ethical protocol, with an oral informed consent.

Statistical analyses were performed using Statistical Package for the Social Sciences (IBM SPSS Statistics $22^{\circledR}$ ). The multiple logistic regression model was used to estimate the predictive power of new risk factors. For statistical purposes, low 5-minute Apgar score was $\leq 8$ and LBW was considered as less than 2,500 grams. Kruskal-Wallis and Pearson $\chi^{2}$ test were applied. The possible new risk factors were included in the multiple logistic regression. Two-sided $p$ values $<0.05$ (95\% confidence interval) were considered to be statistically significant.

\section{Results}

The sample had a total of 596 participants (306 female [51.3\%]), aged on average $8.9 \pm 3.76$ years, of whom 298 (50\%) were controls and $298(50 \%)$ were cases. The cases included 178 (59.7\%) RA and 120 (40.3\%) SA.

Five-minute Apgar score had significant differences among the subgroups $\left(\chi^{2}[2, n=555]=10.6 ; p=0.005 ; n p^{2}=\right.$ $0.019)$, with pairwise comparisons showing significant differences between the SA subgroups and control $(p=0.004)$, as well as between the SA subgroups and the RA subgroup $(p=0.031)$ (Tab. I). There was a statistically significantly different mean birthweight among the subgroups $\left(F_{2,579}=5.74 ; p=\right.$ $0.003 ; \eta p^{2}=0.019$ ), with Games-Howell post hoc test showing statistical differences between the SA subgroup and the controls $(p=0.002)$ (Tab. I). In respect to family history of amblyopia, there was a significant association $\left(\chi^{2}[2, n=562]=\right.$ 12.66; $p=0.002$; Cramer $V=0.150$ ), with a statistically significantly greater association with amblyopia diagnosis in a relative with SA (Tab. II). Family history of strabismus also had a significant association with the tested outcome $\left(\chi^{2}[2, n=\right.$ $561]=11.0 ; p=0.004 ;$ Cramer $V=0.140)$, with a statistically significantly greater association with strabismus diagnosis in a relative in the SA subgroup than in the RA subgroup (Tab. II).

The multinomial logistic regression model (Tab. III) contained binary independent variables (familial refractive error during childhood, amblyopia in a relative, strabismus in a relative, 5-minute Apgar score, neonatal ICU admission, neonatal reanimation, high-risk pregnancy) and continuous independent variable (birthweight). Refractive errors were considered. With these variables, the model improved its statistical significance compared to the intercept alone $\left(\chi^{2}[22, n=473]=\right.$ 307; $p<0.001)$ and had goodness of fit $\left(\chi^{2}[922, n=473]=\right.$ $955 ; p=0.22$ ). The set of independent variables included in the model explained $50.8 \%$ (Nagelkerke $\mathrm{R}^{2}$ ) of the variance in amblyopia development. With this model, we found that amblyopia in a relative increased the odds of having SA and RA, in a factor of 2.64 and 2.02, respectively; strabismus in a relative added a 1.43 risk of having SA; and, finally, that 5-minute Apgar score increased the chance of SA and RA, 3.44 and 3.30 times, respectively.

\section{Discussion}

This is the first study that splits different subtypes of functional amblyopia, SA and RA, in the same study. Furthermore, it shows associations between newly considered risk factors and functional amblyopia subtypes, SA or RA, and some of the newly considered risk factors have also a predictive power for functional amblyopia development, SA or RA.

We found that SA is statistically predicted by family history of amblyopia and has a greater association than RA with family history of strabismus. Also, we observe statistically 
TABLE I - Statistical tests for 5-minute Apgar score and birthweight among subgroups

\begin{tabular}{|c|c|c|c|c|c|c|c|c|}
\hline & \multicolumn{3}{|c|}{$\begin{array}{l}\text { Central tendency measurements, mean } \pm \text { SD; median } \\
\text { (interquartile range) }\end{array}$} & \multirow[t]{2}{*}{$\begin{array}{l}\text { Statistical } \\
\text { test }\end{array}$} & \multicolumn{3}{|c|}{$\begin{array}{l}\text { Significance of pairwise } \\
\text { comparisons, } p\end{array}$} & \multirow[t]{2}{*}{$\begin{array}{l}\text { p value } \\
\text { effect size }\end{array}$} \\
\hline & Control & RA & SA & & $\begin{array}{l}\text { SA vs } \\
\text { control }\end{array}$ & $\begin{array}{l}\text { RA vs } \\
\text { control }\end{array}$ & $\begin{array}{l}\text { SA vs } \\
\text { RA }\end{array}$ & \\
\hline $\begin{array}{l}\text { Apgar at } 5 \text { minutes } \\
\text { (range } 1-10 \text { ) }\end{array}$ & $9.83(9.77 ; 9.90) ; 10 \pm 0$ & $\begin{array}{c}9.79(9.69 \\
9.89) ; 10 \pm 0\end{array}$ & $\begin{array}{c}9.57(9.37 ; \\
9.77) ; 10 \pm 0\end{array}$ & $\begin{array}{c}\chi^{2}(2, n=555) \\
=10.6\end{array}$ & 0.004 & NS & 0.031 & $\begin{array}{c}0.005 ; n p^{2}= \\
0.019\end{array}$ \\
\hline Birthweight, g & $3,198(3,125 ; 3,271)$ & $\begin{array}{c}3,122(3,035 \\
3,210)\end{array}$ & $\begin{array}{c}2,961(2,827 \\
3,096)\end{array}$ & $F_{2,579}=5.74$ & 0.002 & NS & NS & $\begin{array}{c}0.003 ; n p^{2}= \\
0.019\end{array}$ \\
\hline
\end{tabular}

$\eta p^{2}=($ small 0.01, medium 0.06, large 0.14); NS = nonsignificant $(p>0.05) ; R A=$ refractive amblyopia; SA = strabismic amblyopia.

TABLE II - Statistical tests for family history of amblyopia and strabismus among subgroups

\begin{tabular}{|c|c|c|c|c|c|c|c|}
\hline & Statistical test & $p$ value & Effect size, cramer v & \multicolumn{4}{|c|}{ Cross-tabulation } \\
\hline Count & & & & 24 & 25 & 25 & 74 \\
\hline$\%$ Within each subgroup & & & & 8.7 & 14.7 & 21.7 & 13.2 \\
\hline$\%$ Of Total & & & & 4.3 & 4.4 & 4.4 & 13.2 \\
\hline$p$ value & & & & $0.0019 *$ & 0.48 & $0.0023^{*}$ & \\
\hline Strabismus in a relative & $\chi^{2}(2, n=561)=11.0$ & 0.004 & 0.140 & & & & \\
\hline Count & & & & 29 & 10 & 21 & 60 \\
\hline$\%$ Within each subgroup & & & & 10.5 & 5.9 & 18.3 & 10.7 \\
\hline$\%$ Of Total & & & & 5.2 & 1.8 & 3.7 & 10.7 \\
\hline
\end{tabular}

* If adjusted, $\mathrm{p}$ value with significance $(<0.0083$ ); Cramer V (small 0.1, medium 0.3, large 0.5 ).

${ }^{+} \mathrm{p}<0.05$.

$\%=$ Proportion $\times 100$

$\mathrm{RA}=$ refractive amblyopia; $\mathrm{SA}=$ strabismic amblyopia.

TABLE III - Multinomial logistic regression model: new risk factors for strabismic and refractive amblyopia

\begin{tabular}{|c|c|c|c|c|c|c|c|c|c|}
\hline \multirow{2}{*}{$\begin{array}{l}\text { Independent variable } \\
\text { Birthweight }\end{array}$} & \multicolumn{2}{|c|}{ B } & \multirow{2}{*}{$\begin{array}{c}\text { SE } \\
<0.0001\end{array}$} & \multirow{2}{*}{$\begin{array}{c}\text { Wald } \\
0.59\end{array}$} & \multirow{2}{*}{$\frac{\mathbf{d f}}{1}$} & \multirow{2}{*}{$\frac{\text { p value }}{0.44}$} & \multirow{2}{*}{$\begin{array}{l}\text { OOR } \\
1.00\end{array}$} & \multicolumn{2}{|c|}{$95 \% \mathrm{Cl}$ for $\mathrm{OR}$} \\
\hline & RA & $<0.001$ & & & & & & 0.999 & 1.00 \\
\hline & SA & $<0.001$ & $<0.0001$ & 2.48 & 1 & 0.12 & 1.00 & 0.999 & 1.00 \\
\hline \multirow[t]{2}{*}{ Neonatal reanimation } & RA & -0.41 & 0.53 & 0.59 & 1 & 0.44 & 0.66 & 0.23 & 1.89 \\
\hline & SA & -0.22 & 0.51 & 1.49 & 1 & 0.70 & 0.82 & 0.30 & 2.22 \\
\hline Neonatal intensive care unit admission & RA & -0.16 & 0.40 & 0.17 & 1 & 0.68 & 0.85 & 0.39 & 1.85 \\
\hline \multirow[t]{2}{*}{ Low Apgar at the fifth minute } & RA & 1.19 & 78 & 3.12 & 1 & 0.077 & 3.30 & 0.88 & 12.4 \\
\hline & SA & 1.24 & 0.67 & 3.37 & 1 & 0.066 & 3.44 & 0.92 & 12.9 \\
\hline \multirow[t]{2}{*}{ Familiar with refractive error in childhood } & RA & -0.46 & 30 & 2.35 & 1 & 0.13 & 0.63 & 0.35 & 1.14 \\
\hline & SA & -0.50 & 0.31 & 2.66 & 1 & 0.10 & 0.61 & 0.33 & 1.11 \\
\hline
\end{tabular}


TABLE III - Continued

\begin{tabular}{lcccccccccc}
\hline Independent variable & & B & & SE & Wald & df & p value & OOR & 95\% Cl for OR \\
\hline Amblyopia in relative & RA & 0.70 & 0.45 & 2.46 & 1 & 0.12 & 2.02 & 0.84 & 4.84 \\
& SA & 0.97 & 0.43 & 5.14 & 1 & 0.023 & 2.64 & 1.14 & 6.10 \\
High-risk pregnancy & RA & -0.43 & 0.38 & 1.25 & 1 & 0.26 & 0.65 & 0.31 & 1.38 \\
& SA & -0.14 & 0.37 & 0.15 & 1 & 0.70 & 0.87 & 0.42 & 1.79 \\
\hline
\end{tabular}

$p$ value statistically significant when $p<0.05$.

$\mathrm{Cl}=$ confidence interval; $\mathrm{OR}=$ odds ratio; $\mathrm{RA}$ = refractive amblyopia; $\mathrm{SA}$ = strabismic amblyopia.

significant differences regarding the 5-minute Apgar score and LBW, with lowest values in the SA subgroup. On the other hand, we find that the 5-minute Apgar score and the family history of amblyopia increase the risk of both SA and RA.

Relating to neonatal background, the results reveal that 5-minute Apgar score was statistically significantly lower in the SA subgroup than in the control or in the RA subgroups. Low 5-minute Apgar score increases the odds of functional amblyopia development in the SA subgroup as well as in the RA subgroup. In accordance with the prognostic power of 5-minute Apgar score already shown in the literature (20), the present study finds another statistically predicted comorbidity. The common definition of low 5-minute Apgar score is 6 or less (21), since it is associated with higher risk of neonatal encephalopathy, caused by peripartum hypoxia and consequent severe ischemia. The present study defined low 5-minute Apgar score as 8 or less to include less severe hypoxia and its effect on visual development. Until now, no study has analyzed the association of 5-minute Apgar score with SA or RA. The SA subgroup has a higher percentage of obstetrician and neonatal complications, such as high-risk pregnancy, low 1-minute Apgar score, neonatal reanimation, and ICU admission. Birthweight is statistically significantly lower in the SA subgroup than in controls, which is expected (15). The lack of significance related to 1-minute Apgar score in the present study is coherent with the literature (22), since low 1-minute Apgar score is not related to a worse prognosis or future neurodevelopmental complications.

Family history is also relevant. Strabismus in a relative is statistically significantly more associated with the SA subgroup than the RA subgroup. Amblyopia in a relative is a statistically significant predictor for SA and reveals a tendency of increased chance for RA development. This had not been proved before and the only study about this relation was done with another methodology (23). Therefore, family history of strabismus is a robust predictor only for strabismus in children and, in the same way, family history of amblyopia is a robust predictor only for amblyopia in children.

The main limitation of the present study is the hospital bias, with controls being selected from the hospital. When the retrospective data were collected, it was not possible to exclude previous amblyopia in patients who had normal BCVA besides the concomitant presence of ARE.

In the future, in order to determine the impact of these risk factors in strabismus development versus in SA development, a comparison study with squinty nonamblyopic controls versus squinty amblyopic cases should be done. Also, a prospective case-control study with controls selection from the general population could be performed to reinforce the findings of this study.

The present work establishes a predictive model for SA and RA development, including family history and some obstetrician/neonatal new risk factors. The division in amblyopia subtypes, SA and RA, gives a new perspective on the risk factors for amblyopia, with family history and some obstetrician/neonatal outcomes appearing to be more relevant in SA. The recognition of neonatal background and family history as possible risk factors for either SA or RA is important for the education and training of health care providers, either in the primary or in secondary health care system. This can result in early referral of at-risk children to an eye care professional.

\section{Disclosures}

Financial support: No financial support was received for this submission.

Conflict of interest: None of the authors has conflict of interest with this submission.

\section{References}

1. Barrett BT, Bradley A, Candy TR. The relationship between anisometropia and amblyopia. Prog Retin Eye Res. 2013;36: 120-158.

2. Tarczy-Hornoch K, Varma R, Cotter SA, et al. Joint Writing Committee for the Multi-Ethnic Pediatric Eye Disease Study and the Baltimore Pediatric Eye Disease Study Groups. Risk factors for decreased visual acuity in preschool children: the multi-ethnic pediatric eye disease and Baltimore pediatric eye disease studies. Ophthalmology. 2011;118(11):2262-2273.

3. Holmes JM, Clarke MP. Amblyopia. Lancet. 2006;367(9519): 1343-1351.

4. Holmes JM. Screening, confirming, and treating amblyopia based on binocularity. JAMA Ophthalmol. 2014;132(7):820-822.

5. Pascual M, Huang J, Maguire MG, Kulp MT, Quinn GE, Ciner E et al. Risk factors for amblyopia in the Vision in Preschoolers Study. Ophthalmology. 2014;121(3):622-629. e621.

6. Attebo K, Mitchell P, Cumming R, Smith W, Jolly N, Sparkes R. Prevalence and causes of amblyopia in an adult population. Ophthalmology. 1998;105(1):154-159.

7. van Leeuwen R, Eijkemans MJ, Vingerling JR, Hofman $A$, de Jong PT, Simonsz HJ. Risk of bilateral visual impairment in individuals with amblyopia: the Rotterdam study. Br J Ophthalmol. 2007;91(11):1450-1451.

8. Solebo AL, Cumberland PM, Rahi JS. Whole-population vision screening in children aged 45 years to detect amblyopia. Lancet. 2015;385(9984):2308-2319.

9. Solebo AL, Rahi JS. Vision screening in children: why and how? Ophthalmic Epidemiol. 2014;21(4):207-209. 
10. Carlton J, Karnon J, Czoski-Murray C, Smith K, Marr J. The clinical effectiveness and cost-effectiveness of screening programmes for amblyopia and strabismus in children up to the age of 4-5 years: a systematic review and economic evaluation. Health Technol Assess. 2008;12(25):III, XI-194.

11. Mathers $M$, Keyes $M$, Wright $M$. A review of the evidence on the effectiveness of childrens vision screening. Child Care Health Dev. 2010;36(6):756-780.

12. Backman H. Children at risk of developing amblyopia: When to refer for an eye examination. Paediatr Child Health. 2004;9(9): 635-637.

13. Brémond-Gignac D, Copin H, Lapillonne A, Milazzo S; European Network of Study and Research in Eye Development. Visual development in infants: physiological and pathological mechanisms. Curr Opin Ophthalmol. 2011;22(Suppl):S1-S8.

14. Robaei D, Rose KA, Ojaimi E, Kifley A, Martin FJ, Mitchell P. Causes and associations of amblyopia in a population-based sample of 6-year-old Australian children. Arch Ophthalmol. 2006;124(6):878-884.

15. Pai AS-I, Rose KA, Leone JF, et al. Amblyopia prevalence and risk factors in Australian preschool children. Ophthalmology. 2012;119(1):138-144.

16. Boskabadi H, Ashrafzadeh F, Doosti H, Zakerihamidi M. Assessment of risk factors and prognosis in asphyxiated infants. Iranian journal of pediatrics. 2015;25(4):e2006.
17. Abrahamsson M, Magnusson G, Sjöstrand J. Inheritance of strabismus and the gain of using heredity to determine populations at risk of developing strabismus. Acta Ophthalmol Scand. 1999;77(6):653-657.

18. Hammond CJ, Snieder H, Gilbert CE, Spector TD. Genes and environment in refractive error: the twin eye study. Invest Ophthalmol Vis Sci. 2001;42(6):1232-1236.

19. American Academy of Ophthalmology, AAO Pediatric Ophtalmology/Strabismus Panel. San Francisco. Preferred Practice Pattern Guidelines: Amblyopia. September 2012.

20. Casey BM, McIntire DD, Leveno KJ. The continuing value of the Apgar score for the assessment of newborn infants. N Engl J Med. 2001;344(7):467-471.

21. American Academy of Pediatrics Committee on Fetus and Newborn, American College of Obstetricians and Gynecologists Committee on Obstetric Practice. The Apgar Score. Pediatrics. 2015;136(4):819-822.

22. Gynecology. ACoG. The Apgar Score. Committee Opinion. Pediatrics. 2015.

23. Williams C, Northstone K, Howard M, Harvey I, Harrad RA, Sparrow JM. Prevalence and risk factors for common vision problems in children: data from the ALSPAC study. Br J Ophthalmol. 2008;92(7):959-964. 